\title{
Asymptotic Behavior of a Second-Order Fuzzy Rational Difference Equation
}

\author{
Q. Din \\ Department of Mathematics, University of Poonch Rawalakot, Rawalakot 12350, Pakistan \\ Correspondence should be addressed to Q. Din; qamar.sms@gmail.com
}

Received 4 August 2014; Accepted 1 April 2015

Academic Editor: Hjalmar Rosengren

Copyright (C) 2015 Q. Din. This is an open access article distributed under the Creative Commons Attribution License, which permits unrestricted use, distribution, and reproduction in any medium, provided the original work is properly cited.

We study the qualitative behavior of the positive solutions of a second-order rational fuzzy difference equation with initial conditions being positive fuzzy numbers, and parameters are positive fuzzy numbers. More precisely, we investigate existence of positive solutions, boundedness and persistence, and stability analysis of a second-order fuzzy rational difference equation. Some numerical examples are given to verify our theoretical results.

\section{Introduction and Preliminaries}

Recently, studying the qualitative behavior of difference equations and systems is a topic of a great interest. Applications of discrete dynamical systems and difference equations have appeared recently in many areas such as ecology, population dynamics, queuing problems, statistical problems, stochastic time series, combinatorial analysis, number theory, geometry, electrical networks, neural networks, quanta in radiation, genetics in biology, economics, psychology, sociology, physics, engineering, economics, probability theory, and resource management. Unfortunately, these are only considered as the discrete analogs of differential equations. It is a well-known fact that difference equations appeared much earlier than differential equations and were instrumental in paving the way for the development of the latter. It is only recently that difference equations have started receiving the attention they deserve. Perhaps this is largely due to the advent of computers where differential equations are solved by using their approximate difference equation formulations. The theory of discrete dynamical systems and difference equations developed greatly during the last twenty-five years of the twentieth century. The theory of difference equations occupies a central position in applicable analysis. There is no doubt that the theory of difference equations will continue to play an important role in mathematics as a whole.
Nonlinear difference equations of order greater than one are of paramount importance in applications. It is very interesting to investigate the behavior of solutions of a system of higher-order rational difference equations and to discuss the local asymptotic stability of their equilibrium points. Systems of rational difference equations have been studied by several authors. In particular, there has been a great interest in the study of the attractivity of the solutions of such systems. For some interesting results related to the qualitative behavior of the systems of nonlinear difference equations, we refer the interested reader to [1-4].

It is an interesting mathematical problem to investigate the dynamics of nonlinear fuzzy difference equations. For basic theory of fuzzy difference equations we refer to [5]. For qualitative behavior of fuzzy difference equations, one can see [6-11].

Xiao and Shi [12] studied the qualitative behavior of a rational difference equation of the form

$$
y_{n+1}=\frac{y_{n}+y_{n-1}}{p+y_{n} y_{n-1}}
$$

where the parameter $p$ and initial conditions $y_{-1}, y_{0}$ are positive real numbers. 
Our aim in this paper is to investigate the qualitative behavior of following second-order fuzzy rational difference equation:

$$
x_{n+1}=\frac{x_{n}+x_{n-1}}{P+x_{n} x_{n-1}},
$$

where $P$ is positive fuzzy number and initial conditions $x_{-1}$, $x_{0}$ are positive fuzzy numbers.

For further study of (2), we need some basic definitions related to fuzzy difference equations. For more details, we refer to [5-11].

Definition 1. Let $X$ be a normed space. One will denote by $\mathbb{E}(X)$ the family of all fuzzy sets $u: X \rightarrow[0,1]$ which have the following properties:

(i) $u$ is normal; that is, there exists an $x$ in $X$ such that $u(x)=1$,

(ii) $u$ is fuzzy convex; that is, $\min \left\{u\left(x_{1}\right), u\left(x_{2}\right)\right\} \leq u\left(t x_{1}+\right.$ $\left.(1-t) x_{2}\right)$ for all $t \in[0,1]$, and $x_{1}, x_{2} \in X$,

(iii) $u$ is upper semicontinuous,

(iv) the support of $u, \operatorname{supp} u=\overline{\bigcup_{\alpha \in(0,1]}[u]_{\alpha}}=$ $\overline{\{x: u(x)>\alpha\}}$, is compact.

An element $u \in \mathbb{E}(X)$ is called a fuzzy vector. If $X=\mathbb{R}$, then an element $u \in \mathbb{E}(\mathbb{R})$ is called fuzzy number.

Remark 2. In particular, if $\left\{\left[a_{\alpha}, b_{\alpha}\right]\right\}_{\alpha \in(0,1]}$ is a family of compact intervals in $\mathbb{R}$ such that

(i) $\left[a_{\beta}, b_{\beta}\right] \subset\left[a_{\alpha}, b_{\alpha}\right]$ for all $0<\alpha \leq \beta$,

(ii) $\left[\lim _{m \rightarrow \infty} a_{\alpha_{m}}, \lim _{m \rightarrow \infty} b_{\alpha_{m}}\right]=\left[a_{\alpha}, b_{\alpha}\right]$ whenever $\left\{\alpha_{m}\right\} \subset(0,1]$ is an increasing sequence such that $\lim _{m \rightarrow \infty} \alpha_{m}=\alpha$ for $m \geq 1$, then the family $\left\{\left[a_{\alpha}, b_{\alpha}\right]\right\}_{\alpha \in(0,1]}$ represents the $\alpha$-level sets for the fuzzy number $u: \mathbb{R} \rightarrow[0,1]$, defined by

$$
u(x)=\sup \left\{\alpha \in[0,1]: x \in\left[a_{\alpha}, b_{\alpha}\right]\right\} .
$$

Moreover, $\alpha$-cuts of $u$ are given by $[u]_{\alpha}=\{x \in \mathbb{R}$ : $u(x) \geq \alpha\}$ for $\alpha \in[0,1]$. We say a fuzzy number $u$ is positive if supp $u \subset(0, \infty)$. Let $u, v$ be positive fuzzy numbers with $[u]_{\alpha}=\left[u_{l, \alpha}, u_{r, \alpha}\right]$ and $[v]_{\alpha}=\left[v_{l, \alpha}, v_{r, \alpha}\right]$ for $\alpha \in(0,1]$; then we consider the following metric:

$$
D(u, v)=\sup _{\alpha \in(0,1]} \max \left\{\left|u_{l, \alpha}-v_{l, \alpha}\right|,\left|u_{r, \alpha}-v_{r, \alpha}\right|\right\}
$$

A sequence $\left(x_{n}\right)$ of fuzzy numbers is said to be bounded and persists if there exist positive real numbers $\beta, \gamma$ such that $\operatorname{supp} x_{n} \subset[\beta, \gamma]$ for $n=1,2, \ldots$. For more details of boundedness and persistence of fuzzy difference equations, one can see [7-10]. Moreover, if $\left(x_{n}\right)$ is a sequence of positive fuzzy numbers and $x$ is any positive fuzzy number such that $\left[x_{n}\right]_{\alpha}=\left[L_{l, \alpha}, R_{r, \alpha}\right]$ and $[x]_{\alpha}=\left[L_{\alpha}, R_{\alpha}\right]$ for $\alpha \in(0,1]$ and $n=0,1,2, \ldots$, then $\left(x_{n}\right)$ converges to $x$ with respect to metric $D$, if $\lim _{n \rightarrow \infty} D\left(x_{n}, x\right)=0$.
Definition 3. Let $\left(x_{n}\right)$ be a positive solution of (2) and let $\bar{x}$ be its equilibrium point; that is, $\bar{x}=2 \bar{x} /\left(P+\bar{x}^{2}\right)$; then

(i) $\bar{x}$ is stable, if for given $\varepsilon>0$ there exists $\delta=\delta(\varepsilon)>$ 0 such that if $D\left(x_{-i}, \bar{x}\right) \leq \delta$ for $i \in\{-1,0\}$, then $D\left(x_{n}, \bar{x}\right) \leq \varepsilon$ for all $n>0$;

(ii) $\bar{x}$ is said to be unstable if it is not stable;

(iii) $\bar{x}$ is locally asymptotically stable, if it is stable and $\lim _{n \rightarrow \infty} D\left(x_{n}, \bar{x}\right)=0 ;$

(iv) $\bar{x}$ is said to be an attractor if $\lim _{n \rightarrow \infty} D\left(x_{n}, \bar{x}\right)=0$;

(v) $\bar{x}$ is globally asymptotically stable, if it is an attractor and locally asymptotically stable.

\section{Main Results}

Arguing as in $[8,11]$ we have the following theorem for the existence of positive solutions of (2).

Theorem 4. Let $P$ be a positive fuzzy number; then (2) has unique positive solution $\left\{x_{n}\right\}$ with initial conditions $x_{-1}, x_{0}$ being positive fuzzy numbers.

Proof. Let $\left\{x_{n}\right\}$ be a positive solution of (2). The $\alpha$-cuts of positive fuzzy numbers $x_{n}$ and $P$ are given by

$$
\left[x_{n}\right]_{\alpha}=\left[L_{n, \alpha}, R_{n, \alpha}\right], \quad[P]_{\alpha}=\left[P_{l, \alpha}, P_{r, \alpha}\right] \text {. }
$$

Then, from (2), one has

$$
\begin{aligned}
{\left[x_{n+1}\right]_{\alpha} } & =\left[L_{n+1, \alpha}, R_{n+1, \alpha}\right] \\
& =\left[\frac{x_{n}+x_{n-1}}{P+x_{n-1} x_{n}}\right]_{\alpha} \\
& =\frac{\left[x_{n}\right]_{\alpha}+\left[x_{n-1}\right]_{\alpha}}{[P]_{\alpha}+\left[x_{n-1}\right]_{\alpha}\left[x_{n}\right]_{\alpha}} \\
& =\frac{\left[L_{n, \alpha}, R_{n, \alpha}\right]+\left[L_{n-1, \alpha}, R_{n-1, \alpha}\right]}{\left[P_{l, \alpha}, P_{r, \alpha}\right]+\left[L_{n-1, \alpha}, R_{n-1, \alpha}\right]\left[L_{n, \alpha}, R_{n, \alpha}\right]} \\
& =\left[\frac{L_{n, \alpha}+L_{n-1, \alpha}}{P_{r, \alpha}+R_{n-1, \alpha} R_{n, \alpha}}, \frac{R_{n, \alpha}+R_{n-1, \alpha}}{P_{l, \alpha}+L_{n-1, \alpha} L_{n, \alpha}}\right] .
\end{aligned}
$$

Hence, one has the following system:

$$
\begin{aligned}
L_{n+1, \alpha} & =\frac{L_{n, \alpha}+L_{n-1, \alpha}}{P_{r, \alpha}+R_{n-1, \alpha} R_{n, \alpha}}, \\
R_{n+1, \alpha} & =\frac{R_{n, \alpha}+R_{n-1, \alpha}}{P_{l, \alpha}+L_{n-1, \alpha} L_{n, \alpha}},
\end{aligned}
$$

for $n=0,1,2, \ldots$, and $\alpha \in(0,1]$. Let $\left(L_{n, \alpha}, R_{n, \alpha}\right)$ be a solution of system (7) with initial conditions $L_{-1, \alpha}, L_{0, \alpha}, R_{-1, \alpha}, R_{0, \alpha}$ for every $\alpha \in(0,1]$. Then, it is sufficient to prove that $\left(x_{n}\right)$ is a solution of our original system (2) with initial conditions $x_{-1}, x_{0}$ and $\left[x_{n}\right]_{\alpha}=\left[L_{n, \alpha}, R_{n, \alpha}\right]$ for every $n=0,1,2, \ldots$ and $\alpha \in(0,1]$. Moreover, for any fuzzy number $u \in \mathbb{E}(\mathbb{R})$ with $[u]_{\alpha}=[\underline{u}(\alpha), \bar{u}(\alpha)]$, one has $\underline{u}(\alpha)$ that is nondecreasing and left continuous and $\bar{u}(\alpha)$ that is nonincreasing and left 
continuous for $0<\alpha \leq 1$. As $A, x_{-1}, x_{0}$ are positive fuzzy numbers, for $\alpha_{1}, \alpha_{2} \in(0,1]$ with $\alpha_{1} \leq \alpha_{2}$, one has

$$
\begin{gathered}
0<A_{l, \alpha_{1}} \leq A_{l, \alpha_{2}} \leq A_{r, \alpha_{2}} \leq A_{r, \alpha_{1}}, \\
0<B_{l, \alpha_{1}} \leq B_{l, \alpha_{2}} \leq B_{r, \alpha_{2}} \leq B_{r, \alpha_{1}}, \\
0<L_{-1, \alpha_{1}} \leq L_{-1, \alpha_{2}} \leq R_{-1, \alpha_{2}} \leq R_{-1, \alpha_{1}}, \\
0<L_{0, \alpha_{1}} \leq L_{0, \alpha_{2}} \leq R_{0, \alpha_{2}} \leq R_{0, \alpha_{1}} .
\end{gathered}
$$

Now, it is easy to show by induction on $n$ that

$$
0<L_{n, \alpha_{1}} \leq L_{n, \alpha_{2}} \leq R_{n, \alpha_{2}} \leq R_{n, \alpha_{1}}, \quad n=-1,0,1,2, \ldots
$$

Similarly, by induction on $n$, one can show that $L_{n, \alpha}$, $R_{n, \alpha}$ are left continuous for all $n=1,2, \ldots$, and $0<$ $\alpha \leq 1$. Furthermore, it can be inductively proved that $\overline{\bigcup_{\alpha \in(0,1]}\left[L_{n, \alpha}, R_{n, \alpha}\right]} \subset(0, \infty)$; that is, $\overline{\bigcup_{\alpha \in(0,1]}\left[L_{n, \alpha}, R_{n, \alpha}\right]}$ is compact. Hence, $\left[L_{n, \alpha}, R_{n, \alpha}\right]$ determines a sequence $\left(x_{n}\right)$ of positive fuzzy numbers such that $\left[x_{n}\right]_{\alpha}=\left[L_{n, \alpha}, R_{n, \alpha}\right]$ for every $n=0,1,2, \ldots$ and $\alpha \in(0,1]$.

In order to study the further dynamics of the fuzzy difference equation (2) we will use the results concerning the behavior of the solutions of the corresponding system of two parametric ordinary difference equations given by

$$
u_{n+1}=\frac{u_{n}+u_{n-1}}{a_{1}+v_{n-1} v_{n}}, \quad v_{n+1}=\frac{v_{n}+v_{n-1}}{a_{2}+u_{n-1} u_{n}} \text {, }
$$

where the parameters $a_{1}, a_{2}$ are positive real numbers, and initial conditions $u_{-1}, u_{0}, v_{-1}, v_{0}$ are positive real numbers.

Let $a_{1}<2, a_{2}<2$; then $O=(0,0)$ and $E=$ $\left(\sqrt{2-a_{2}}, \sqrt{2-a_{1}}\right)$ are equilibrium points of system (10). Moreover, $E$ is unique positive equilibrium of (10).

Theorem 5. Let $\left\{\left(u_{n}, v_{n}\right)\right\}_{n=-1}^{\infty}$ be any solution of system (10); then the following statements are true.

(1) For every $n \geq 1$ the following results hold:

$$
\begin{aligned}
& 0 \leq u_{n} \leq u_{-1}\left(\frac{1-\sqrt{4 a_{1}+1}}{2 a_{1}}\right)^{n}+u_{0}\left(\frac{\sqrt{4 a_{1}+1}+1}{2 a_{1}}\right)^{n}, \\
& 0 \leq v_{n} \leq v_{-1}\left(\frac{1-\sqrt{4 a_{2}+1}}{2 a_{2}}\right)^{n}+v_{0}\left(\frac{\sqrt{4 a_{2}+1}+1}{2 a_{2}}\right)^{n},
\end{aligned}
$$

with initial conditions $u_{-1}, u_{0}, v_{-1}, v_{0}$.

(2) For the equilibrium point $O=(0,0)$ of system (10) the following results hold.

(i) Let $2<a_{1}$ and $2<a_{2}$; then equilibrium point $\mathrm{O}=(0,0)$ of system $(10)$ is locally asymptotically stable.

(ii) If $2>a_{1}$ or $2>a_{2}$, then equilibrium point $O=$ $(0,0)$ of system $(10)$ is unstable.
(3) If $2>a_{1}$ and $2>a_{2}$, then the unique positive equilibrium point $E=\left(\sqrt{2-a_{2}}, \sqrt{2-a_{1}}\right)$ of system (10) is unstable.

(4) Let $2<a_{1}$ and $2<a_{2}$; then the equilibrium point $\mathrm{O}=$ $(0,0)$ of system $(10)$ is globally asymptotically stable.

Proof. (1) It follows from (10) that

$$
u_{n+1} \leq \frac{1}{a_{1}}\left(u_{n-1}+u_{n}\right), \quad v_{n+1} \leq \frac{1}{a_{2}}\left(v_{n-1}+v_{n}\right),
$$

for all $n=0,1, \ldots$. Moreover, consider the following secondorder linear difference equations:

$$
x_{n+1}=\frac{1}{a_{1}}\left(x_{n-1}+x_{n}\right), \quad y_{n+1}=\frac{1}{a_{2}}\left(y_{n-1}+y_{n}\right) .
$$

The solutions of these equations are given by

$$
\begin{aligned}
& x_{n}=c_{1}\left(\frac{1-\sqrt{4 a_{1}+1}}{2 a_{1}}\right)^{n}+c_{2}\left(\frac{\sqrt{4 a_{1}+1}+1}{2 a_{1}}\right)^{n}, \\
& y_{n}=c_{3}\left(\frac{1-\sqrt{4 a_{2}+1}}{2 a_{2}}\right)^{n}+c_{4}\left(\frac{\sqrt{4 a_{2}+1}+1}{2 a_{2}}\right)^{n},
\end{aligned}
$$

for all $n=1,2, \ldots$, where $c_{1}, c_{2}, c_{3}$, and $c_{4}$ depend on initial conditions $x_{-1}, x_{0}, y_{-1}, y_{0}$. Let $x_{-1}=u_{-1}, x_{0}=u_{0}, y_{-1}=v_{-1}$, $y_{0}=v_{0}$; then by comparison we obtain

$$
\begin{aligned}
& 0 \leq u_{n} \leq u_{-1}\left(\frac{1-\sqrt{4 a_{1}+1}}{2 a_{1}}\right)^{n}+u_{0}\left(\frac{\sqrt{4 a_{1}+1}+1}{2 a_{1}}\right)^{n}, \\
& 0 \leq v_{n} \leq v_{-1}\left(\frac{1-\sqrt{4 a_{2}+1}}{2 a_{2}}\right)^{n}+v_{0}\left(\frac{\sqrt{4 a_{2}+1}+1}{2 a_{2}}\right)^{n} .
\end{aligned}
$$

(2) To construct corresponding linearized form of system (10), we consider the following transformation:

$$
\left(u_{n}, u_{n-1}, v_{n}, v_{n-1}\right) \longmapsto\left(f, f_{1}, g, g_{1}\right),
$$

where $f=u_{n+1}, g=v_{n+1}, f_{1}=u_{n}$, and $g_{1}=v_{n}$. The linearized system of (10) about the equilibrium point $(0,0)$ is given by

$$
X_{n+1}=F_{J}(0,0) X_{n}
$$

where

$$
\begin{gathered}
X_{n}=\left(\begin{array}{c}
u_{n} \\
u_{n-1} \\
v_{n} \\
v_{n-1}
\end{array}\right), \\
F_{J}(0,0)=\left(\begin{array}{cccc}
\frac{1}{a_{1}} & \frac{1}{a_{1}} & 0 & 0 \\
1 & 0 & 0 & 0 \\
0 & 0 & \frac{1}{a_{2}} & \frac{1}{a_{2}} \\
0 & 0 & 1 & 0
\end{array}\right) .
\end{gathered}
$$


The characteristic polynomial of $F_{J}(0,0)$ is given by

$$
\begin{aligned}
P(\lambda)= & \lambda^{4}-\left(\frac{1}{a_{1}}+\frac{1}{a_{2}}\right) \lambda^{3}+\left(\frac{1}{a_{1} a_{2}}-\frac{1}{a_{1}}-\frac{1}{a_{2}}\right) \lambda^{2} \\
& +\frac{2}{a_{1} a_{2}} \lambda+\frac{1}{a_{1} a_{2}} .
\end{aligned}
$$

The roots of characteristic polynomial (19) are given by

$$
\lambda_{1,2}=\frac{1 \pm \sqrt{4 a_{1}+1}}{2 a_{1}}, \quad \lambda_{3,4}=\frac{1 \pm \sqrt{4 a_{2}+1}}{2 a_{2}} .
$$

(i) Since all eigenvalues of Jacobian matrix $F_{J}(0,0)$ about $(0,0)$ lie in open unit dick $|\lambda|<1$ if and only if $2<$ $a_{1}$ and $2<a_{2}$, the equilibrium point $(0,0)$ is locally asymptotically stable.

(ii) Similarly, all eigenvalues of Jacobian matrix $F_{J}(0,0)$ about $(0,0)$ lie outside open unit dick $|\lambda|<1$ if and only if $2>a_{1}$ and $2>a_{2}$. Hence, the equilibrium point $(0,0)$ is unstable in this case.

(3) The linearized system of (7) about the equilibrium point $E=\left(\sqrt{2-a_{2}}, \sqrt{2-a_{1}}\right)$ is given by

$$
X_{n+1}=F_{J}(E) X_{n}
$$

where

$$
\begin{gathered}
X_{n}=\left(\begin{array}{c}
u_{n} \\
u_{n-1} \\
v_{n} \\
v_{n-1}
\end{array}\right), \\
F_{J}(E)=\left(\begin{array}{cccc}
\frac{1}{2} & \frac{1}{2} & A & A \\
1 & 0 & 0 & 0 \\
A & A & \frac{1}{2} & \frac{1}{2} \\
0 & 0 & 1 & 0
\end{array}\right),
\end{gathered}
$$

where $A=-(1 / 2) \sqrt{2-a_{1}} \sqrt{2-a_{2}}$. The characteristic polynomial of $F_{J}(E)$ is given by

$$
P(\lambda)=\lambda^{4}-\lambda^{3}-\left(\frac{3}{4}+A^{2}\right) \lambda^{2}+\left(\frac{1}{2}-2 A^{2}\right) \lambda+\frac{1}{4}-A^{2} .
$$

The roots of characteristic polynomial $P(\lambda)(23)$ are given by

$$
\begin{aligned}
& \lambda_{1,2}=\frac{1}{4}\left(1-2 A \pm \sqrt{4 A^{2}-20 A+9}\right), \\
& \lambda_{3,4}=\frac{1}{4}\left(1+2 A \pm \sqrt{4 A^{2}+20 A+9}\right) .
\end{aligned}
$$

It is sufficient to prove that any one of these roots has absolute value greater than one. For this consider

$$
\left|\lambda_{1}\right|=\frac{1}{4}\left(1-2 A+\sqrt{4 A^{2}-20 A+9}\right)>1 .
$$

(4) Assume that $2<a_{1}$ and $2<a_{2}$; then from (i) of (2) of Theorem $5(0,0)$ is locally asymptotically stable. Let $\left\{\left(x_{n}, y_{n}\right)\right\}$ be any positive solution of (10); then it remains to show that $\lim _{n \rightarrow \infty} u_{n}=0$ and $\lim _{n \rightarrow \infty} v_{n}=0$. Moreover, $\left(\sqrt{4 a_{1}+1}+\right.$ 1) $/ 2 a_{1}<1$ and $\left(\sqrt{4 a_{2}+1}+1\right) / 2 a_{2}<1$ if and only if $2<a_{1}$ and $2<a_{2}$, respectively. It follows from (1) of Theorem 5 that

$$
\begin{aligned}
0 \leq & u_{n} \leq u_{-1}\left(\frac{1-\sqrt{4 a_{1}+1}}{2 a_{1}}\right)^{n} \\
& +u_{0}\left(\frac{\sqrt{4 a_{1}+1}+1}{2 a_{1}}\right)^{n} \rightarrow 0 \text { as } n \rightarrow \infty, \\
0 \leq v_{n} \leq v_{-1}\left(\frac{1-\sqrt{4 a_{2}+1}}{2 a_{2}}\right)^{n} & \\
& +v_{0}\left(\frac{\sqrt{4 a_{2}+1}+1}{2 a_{2}}\right)^{n} \rightarrow 0 \text { as } n \rightarrow \infty .
\end{aligned}
$$

Hence, $\lim _{n \rightarrow \infty} u_{n}=\lim _{n \rightarrow \infty} v_{n}=0$.

Next, we discuss the rate of convergence of positive solutions of system (10) which converge to equilibrium point $(0,0)$ of this system. Similar methods can be found in [1-4]. The following result gives the rate of convergence of solutions of a system of difference equations

$$
X_{n+1}=(A+B(n)) X_{n}
$$

where $X_{n}$ is an $m$-dimensional vector, $A \in C^{m \times m}$ is a constant matrix, and $B: \mathbb{Z}^{+} \rightarrow C^{m \times m}$ is a matrix function satisfying

$$
\|B(n)\| \longrightarrow 0
$$

as $n \rightarrow \infty$, where $\|\cdot\|$ denotes any matrix norm which is associated with the vector norm

$$
\|(x, y)\|=\sqrt{x^{2}+y^{2}} .
$$

Proposition 6 (Perron's theorem; see [13]). Suppose that condition (28) holds. If $X_{n}$ is a solution of (27), then either $X_{n}=0$ for all large $n$ or

$$
\rho=\lim _{n \rightarrow \infty}\left(\left\|X_{n}\right\|\right)^{1 / n}
$$

exists and is equal to the modulus of one the eigenvalues of matrix A.

Proposition 7 (see [13]). Suppose that condition (28) holds. If $X_{n}$ is a solution of (27), then either $X_{n}=0$ for all large $n$ or

$$
\rho=\lim _{n \rightarrow \infty} \frac{\left\|X_{n+1}\right\|}{\left\|X_{n}\right\|}
$$

exists and is equal to the modulus of one the eigenvalues of matrix A. 
Let $\left\{\left(u_{n}, v_{n}\right)\right\}$ be any solution of system (10) such that $\lim _{n \rightarrow \infty} u_{n}=\bar{u}$ and $\lim _{n \rightarrow \infty} v_{n}=\bar{v}$, where $(\bar{u}, \bar{v})=(0,0)$. To find the error terms, one has from system (10)

$$
\begin{aligned}
& u_{n+1}-\bar{u}=\frac{u_{n}+u_{n-1}}{a_{1}+v_{n} v_{n-1}}-\frac{2 \bar{u}}{a_{1}+\bar{v}^{2}} \\
& =\frac{u_{n}-\bar{u}}{a_{1}+v_{n} v_{n-1}}+\frac{u_{n-1}-\bar{u}}{a_{1}+v_{n} v_{n-1}} \\
& -\frac{2 \bar{u} v_{n-1}\left(v_{n}-\bar{v}\right)}{\left(a_{1}+v_{n} v_{n-1}\right)\left(a_{1}+\bar{v}^{2}\right)} \\
& -\frac{2 \bar{u} \bar{v}\left(v_{n-1}-\bar{v}\right)}{\left(a_{1}+v_{n} v_{n-1}\right)\left(a_{1}+\bar{v}^{2}\right)} \\
& =a_{n}\left(u_{n}-\bar{u}\right)+b_{n}\left(u_{n-1}-\bar{u}\right) \\
& +c_{n}\left(v_{n}-\bar{v}\right)+d_{n}\left(v_{n-1}-\bar{v}\right), \\
& v_{n+1}-\bar{v}=\frac{v_{n}+v_{n-1}}{a_{2}+u_{n} u_{n-1}}-\frac{2 \bar{v}}{a_{2}+\bar{u}^{2}} \\
& =-\frac{2 \bar{v} u_{n-1}\left(u_{n}-\bar{u}\right)}{\left(a_{2}+u_{n} u_{n-1}\right)\left(a_{2}+\bar{u}^{2}\right)} \\
& -\frac{2 \bar{u} v\left(u_{n-1}-\bar{u}\right)}{\left(a_{2}+u_{n} u_{n-1}\right)\left(a_{2}+\bar{u}^{2}\right)} \\
& +\frac{v_{n}-\bar{v}}{a_{2}+u_{n} u_{n-1}}+\frac{v_{n-1}-\bar{v}}{a_{2}+u_{n} u_{n-1}} \\
& =a_{n}^{1}\left(u_{n}-\bar{u}\right)+b_{n}^{1}\left(u_{n-1}-\bar{u}\right) \\
& +c_{n}^{1}\left(v_{n}-\bar{v}\right)+d_{n}^{1}\left(v_{n-1}-\bar{v}\right) \text {. }
\end{aligned}
$$

Let $e_{n}^{1}=u_{n}-\bar{u}$, and $e_{n}^{2}=v_{n}-\bar{v}$; then one has

$$
\begin{aligned}
& e_{n+1}^{1}=a_{n} e_{n}^{1}+b_{n} e_{n-1}^{1}+c_{n} e_{n}^{2}+d_{n} e_{n-1}^{2}, \\
& e_{n+1}^{2}=a_{n}^{1} e_{n}^{1}+b_{n}^{1} e_{n-1}^{1}+c_{n}^{1} e_{n}^{2}+d_{n}^{1} e_{n-1}^{2},
\end{aligned}
$$

where

$$
\begin{gathered}
a_{n}=b_{n}=\frac{1}{a_{1}+v_{n} v_{n-1}}, \\
c_{n}=-\frac{2 \bar{u} v_{n-1}}{\left(a_{1}+v_{n} v_{n-1}\right)\left(a_{1}+\bar{v}^{2}\right)}, \\
d_{n}=-\frac{2 \overline{u v}}{\left(a_{1}+v_{n} v_{n-1}\right)\left(a_{1}+\bar{v}^{2}\right)}, \\
a_{n}^{1}=-\frac{2 \bar{v} u_{n-1}}{\left(a_{2}+u_{n} u_{n-1}\right)\left(a_{2}+\bar{u}^{2}\right)},
\end{gathered}
$$

$$
\begin{gathered}
b_{n}^{1}=-\frac{2 \overline{u v}}{\left(a_{2}+u_{n} u_{n-1}\right)\left(a_{2}+\bar{u}^{2}\right)} \\
c_{n}^{1}=d_{n}^{1}=\frac{1}{a_{2}+u_{n} u_{n-1}} .
\end{gathered}
$$

Moreover, we obtain

$$
\begin{gathered}
\lim _{n \rightarrow \infty} a_{n}=\lim _{n \rightarrow \infty} b_{n}=\frac{1}{a_{1}+\bar{v}^{2}}, \\
\lim _{n \rightarrow \infty} c_{n}=\lim _{n \rightarrow \infty} d_{n}=-\frac{2 \overline{u v}}{\left(a_{1}+\bar{v}^{2}\right)^{2}}, \\
\lim _{n \rightarrow \infty} a_{n}^{1}=\lim _{n \rightarrow \infty} b_{n}^{1}=-\frac{2 \overline{u v}}{\left(a_{2}+\bar{u}^{2}\right)^{2}}, \\
\lim _{n \rightarrow \infty} c_{n}^{1}=\lim _{n \rightarrow \infty} d_{n}^{1}=\frac{1}{a_{2}+\bar{u}^{2}} .
\end{gathered}
$$

Now the limiting system of error terms about $(\bar{u}, \bar{v})=(0,0)$ can be written as

$$
\left[\begin{array}{c}
e_{n+1}^{1} \\
e_{n}^{1} \\
e_{n+1}^{2} \\
e_{n}^{2}
\end{array}\right]=\left[\begin{array}{cccc}
\frac{1}{a_{1}} & \frac{1}{a_{1}} & 0 & 0 \\
1 & 0 & 0 & 0 \\
0 & 0 & \frac{1}{a_{2}} & \frac{1}{a_{2}} \\
0 & 0 & 1 & 0
\end{array}\right]\left[\begin{array}{c}
e_{n}^{1} \\
e_{n-1}^{1} \\
e_{n}^{2} \\
e_{n-1}^{2}
\end{array}\right],
$$

which is similar to linearized system of (10) about the equilibrium point $(0,0)$.

Using Proposition 6, one has following result.

Theorem 8. Assume that $\left\{\left(u_{n}, v_{n}\right)\right\}$ is a positive solution of system (7) such that $\lim _{n \rightarrow \infty} u_{n}=0$ and $\lim _{n \rightarrow \infty} v_{n}=0$. Then, the error vector $E_{n}=\left[\begin{array}{c}e_{n}^{1} \\ e_{n-1}^{1} \\ e_{n}^{2} \\ e_{n-1}^{2}\end{array}\right]$ of every solution of (10) satisfies both of the following asymptotic relations:

$$
\begin{gathered}
\lim _{n \rightarrow \infty}\left(\left\|E_{n}\right\|\right)^{1 / n}=\left|\lambda F_{J}(0,0)\right|, \\
\lim _{n \rightarrow \infty} \frac{\left\|E_{n+1}\right\|}{\left\|E_{n}\right\|}=\left|\lambda F_{J}(0,0)\right|,
\end{gathered}
$$

where $\lambda F_{J}(0,0)$ are the characteristic roots of Jacobian matrix $F_{J}(0,0)$.

The following theorem is similar to Theorem 2.5 of [11].

Theorem 9. Assume that $\left(x_{n}\right)$ is a positive solution of (2) and $2<P_{l, \alpha}$; then $\left(x_{n}\right)$ is bounded and persists. Moreover, $\left(x_{n}\right)$ converges to 0 . 
Proof. Consider the following system:

$$
s_{n+1}=\frac{s_{n}+s_{n-1}}{\gamma_{P}+t_{n-1} t_{n}}, \quad t_{n+1}=\frac{t_{n}+t_{n-1}}{\beta_{P}+s_{n-1} s_{n}},
$$

where

$$
\left[P_{l, \alpha}, P_{r, \alpha}\right] \subset \overline{\bigcup_{\alpha \in(0,1]}\left[P_{l, \alpha}, P_{r, \alpha}\right]} \subset\left[\beta_{P}, \gamma_{P}\right]
$$

Let $\left(s_{n}, t_{n}\right)$ be a solution of system (38) with initial conditions $s_{-1}=\beta_{-1}, s_{0}=\beta_{0}, t_{-1}=\gamma_{-1}, t_{0}=\gamma_{0}$, where $\beta_{i}, \gamma_{i}$ for $i \epsilon$ $\{-1,0\}$ are given as

$$
\begin{gathered}
{\left[L_{-l, \alpha}, R_{-1, \alpha}\right] \subset \overline{\bigcup_{\alpha \in(0,1]}\left[L_{-1, \alpha}, R_{-1, \alpha}\right]} \subset\left[\beta_{-1}, \gamma_{-1}\right],} \\
{\left[L_{0, \alpha}, R_{0, \alpha}\right] \subset \overline{\bigcup_{\alpha \in(0,1]}\left[L_{0, \alpha}, R_{0, \alpha}\right]} \subset\left[\beta_{0}, \gamma_{0}\right] .}
\end{gathered}
$$

Then, it follows that

$$
s_{1}=\frac{s_{0}+s_{-1}}{\gamma_{A}+t_{-1} t_{0}} \leq L_{1, \alpha}, \quad t_{1}=\frac{t_{0}+t_{-1}}{\beta_{A}+s_{-1} s_{0}} \geq R_{1, \alpha} .
$$

Hence, by induction one has $s_{n} \leq L_{n, \alpha}, t_{n} \geq R_{n, \alpha}$ for all $n=$ $1,2, \ldots$. Assume that $2<P_{l, \alpha}$; then it follows that $2<\gamma_{A}, 2<$ $\beta_{A}$. Hence, the solution $\left(s_{n}, t_{n}\right)$ of system (38) is bounded and persists, and so is the solution $\left(x_{n}\right)$ of (2). Next, from (4) of Theorem 5, it is easy to see that $\lim _{n \rightarrow \infty} L_{n, \alpha}=\lim _{n \rightarrow \infty} R_{n, \alpha}=$ 0 . Then, it follows that

$$
\begin{aligned}
& \lim _{n \rightarrow \infty} D\left(x_{n}, 0\right) \\
& \quad=\lim _{n \rightarrow \infty} \sup _{\alpha \in(0,1]}\left\{\max \left\{\left|L_{n, \alpha}-0\right|,\left|R_{n, \alpha}-0\right|\right\}\right\}=0 .
\end{aligned}
$$

Theorem 10. Assume that $P$ is a positive real number and $P<$ 2; then unique positive equilibrium point $\bar{x}=\sqrt{2-P}$ of (2) is unstable.

Proof. Let $\left(x_{n}\right)$ be a positive solution of (2) and let $P$ be a positive real number with $P<2$. Then, from (1) of Theorem 5, either $\lim _{n \rightarrow \infty} R_{n, \alpha}=0$ and $\lim _{n \rightarrow \infty} L_{n, \alpha}=\infty$ or $\lim _{n \rightarrow \infty} R_{n, \alpha}=\infty$ and $\lim _{n \rightarrow \infty} L_{n, \alpha}=0$. Hence, there are no positive numbers $\beta, \gamma$ such that $\bigcup_{\alpha \in(0,1]}\left[L_{n, \alpha}, R_{n, \alpha}\right] \subset[\beta, \gamma]$. This completes the proof.

\section{Examples}

Example 1. Consider system (10) with initial conditions $u_{-1}=$ $0.5, u_{0}=0.2, v_{-1}=0.05, v_{0}=0.4$. Moreover, choose the parameters $a_{1}=a_{2}=2.001$. Then, system (10) can be written as

$$
u_{n+1}=\frac{u_{n}+u_{n-1}}{2.001+v_{n-1} v_{n}}, \quad v_{n+1}=\frac{v_{n}+v_{n-1}}{2.001+u_{n-1} u_{n}},
$$

with initial conditions $u_{-1}=0.5, u_{0}=0.2, v_{-1}=0.05$, $v_{0}=0.4$. In this case, $\lim _{n \rightarrow \infty} u_{n}=0$ and $\lim _{n \rightarrow \infty} v_{n}=0$. Moreover, for system (43) the plot of $u_{n}$ is shown in Figure 1 and the plot of $v_{n}$ is shown in Figure 2.

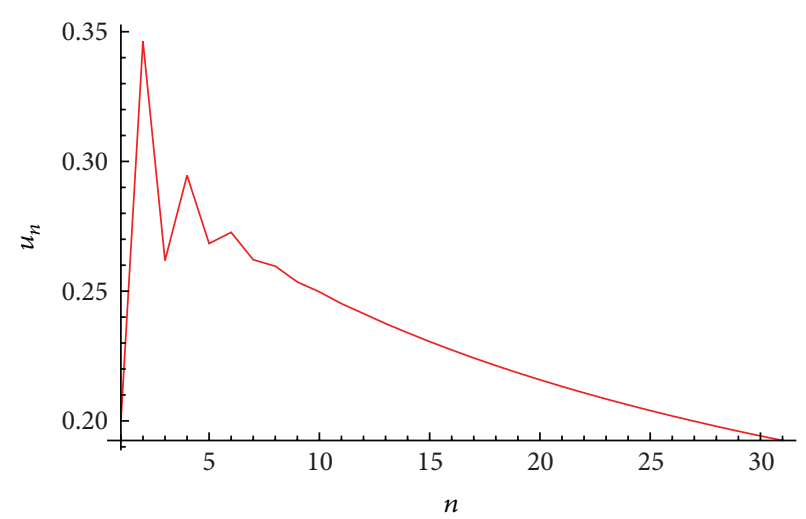

FIgURE 1: Plot of $u_{n}$ for system (43).

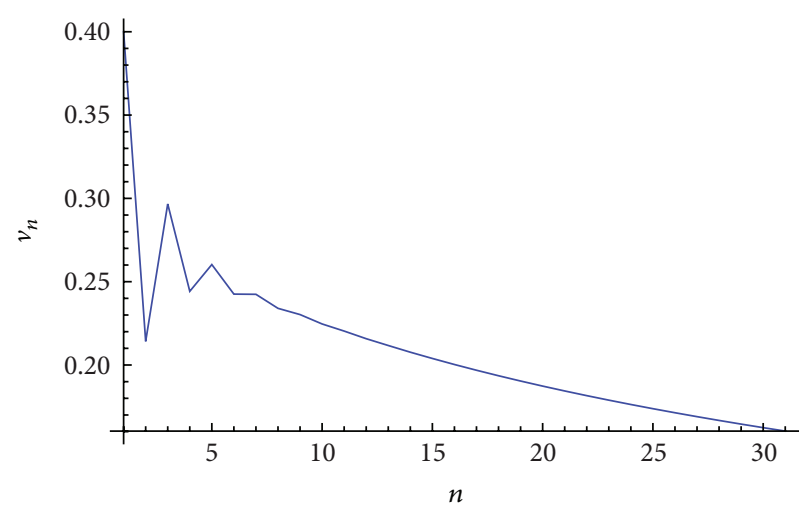

Figure 2: Plot of $v_{n}$ for system (43).

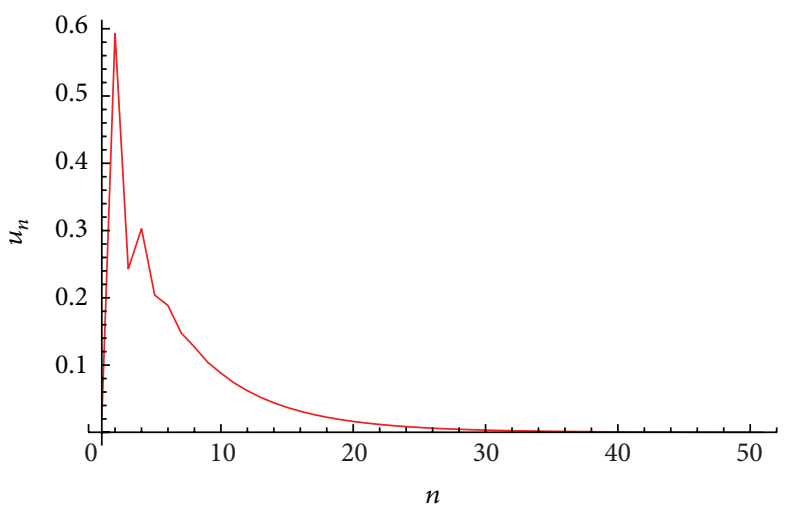

Figure 3: Plot of $u_{n}$ for system (44).

Example 2. Consider system (10) with initial conditions $u_{-1}=1.5, u_{0}=0.02, v_{-1}=1.5, v_{0}=0.04$. Moreover, choose the parameters $a_{1}=2.5, a_{2}=2.1$. Then, system (10) can be written as

$$
u_{n+1}=\frac{u_{n}+u_{n-1}}{2.5+v_{n-1} v_{n}}, \quad v_{n+1}=\frac{v_{n}+v_{n-1}}{2.1+u_{n-1} u_{n}},
$$

with initial conditions $u_{-1}=1.5, u_{0}=0.02, v_{-1}=1.5$, $v_{0}=0.04$. In this case, $\lim _{n \rightarrow \infty} u_{n}=0$ and $\lim _{n \rightarrow \infty} v_{n}=0$. Moreover, for system (44) the plot of $u_{n}$ is shown in Figure 3 and the plot of $v_{n}$ is shown in Figure 4. 


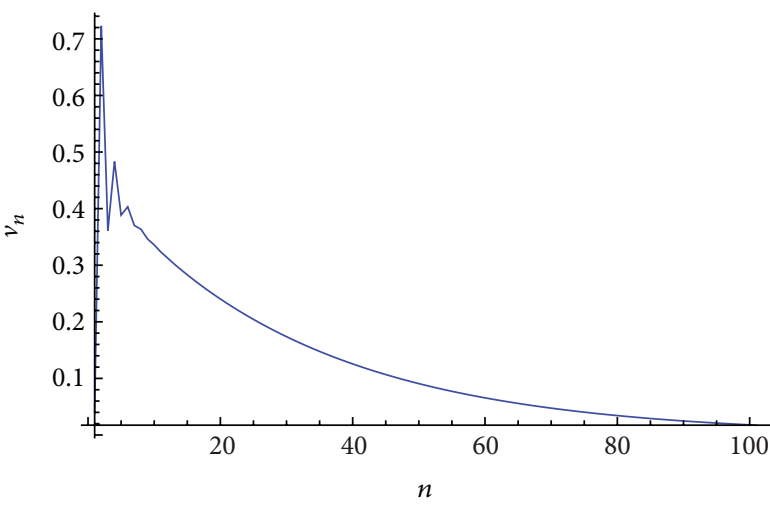

Figure 4: Plot of $v_{n}$ for system (44).

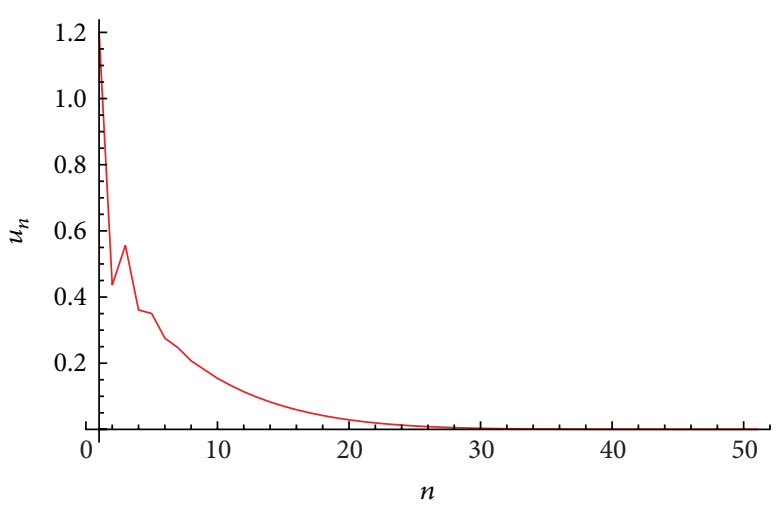

Figure 5: Plot of $u_{n}$ for system (45).

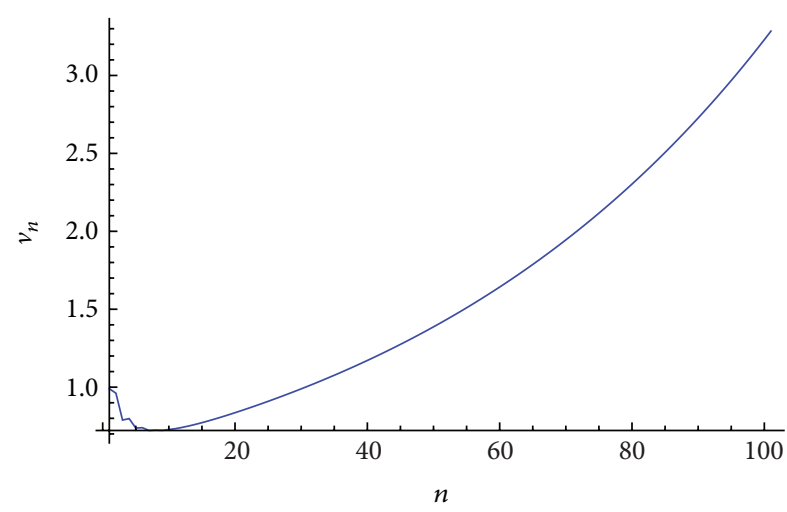

FIgURE 6: Plot of $v_{n}$ for system (45).

Example 3. Consider system (10) with initial conditions $u_{-1}=0.1, u_{0}=1.2, v_{-1}=1, v_{0}=0.99$. Moreover, choose the parameters $a_{1}=1.99, a_{2}=1.95$. Then, system (10) can be written as

$$
u_{n+1}=\frac{u_{n}+u_{n-1}}{1.99+v_{n-1} v_{n}}, \quad v_{n+1}=\frac{v_{n}+v_{n-1}}{1.95+u_{n-1} u_{n}},
$$

with initial conditions $u_{-1}=0.1, u_{0}=1.2, v_{-1}=1, v_{0}=0.99$. In this case $\lim _{n \rightarrow \infty} u_{n}=0$ but $\lim _{n \rightarrow \infty} v_{n}=\infty$. Moreover, for system (45) the plot of $u_{n}$ is shown in Figure 5 and the plot of $v_{n}$ is shown in Figure 6.

\section{Conflict of Interests}

The author declares that he has no conflict of interests regarding the publication of this paper.

\section{Acknowledgment}

This work was supported by the Higher Education Commission of Pakistan.

\section{References}

[1] Q. Din and T. Donchev, "Global character of a host-parasite model," Chaos, Solitons \& Fractals, vol. 54, pp. 1-7, 2013.

[2] Q. Din, "Global stability of a population model," Chaos, Solitons \& Fractals, vol. 59, pp. 119-128, 2014.

[3] Q. Din, T. F. Ibrahim, and K. A. Khan, "Behavior of a competitive system of second-order difference equations," The Scientific World Journal, vol. 2014, Article ID 283982, 9 pages, 2014.

[4] Q. Din, K. A. Khan, and A. Nosheen, "Stability analysis of a system of exponential difference equations," Discrete Dynamics in Nature and Society, vol. 2014, Article ID 375890, 11 pages, 2014.

[5] V. Lakshmikantham and A. S. Vatsala, "Basic theory of fuzzy difference equations," Journal of Difference Equations and Applications, vol. 8, no. 11, pp. 957-968, 2002.

[6] R. C. Bassanezi, L. C. de Barros, and P. A. Tonelli, "Attractors and asymptotic stability for fuzzy dynamical systems," Fuzzy Sets and Systems, vol. 113, no. 3, pp. 473-483, 2000.

[7] G. Papaschinopoulos and B. K. Papadopoulos, "On the fuzzy difference equation $x_{n+1}=A+B / x_{n}$," Soft Computing, vol. 6 , pp. 456-461, 2002.

[8] G. Papaschinopoulos and B. K. Papadopoulos, "On the fuzzy difference equation $x_{n+1}=A+x_{n} / x_{n-m}$," Fuzzy Sets and Systems, vol. 129, no. 1, pp. 73-81, 2002.

[9] G. Papaschinopoulos and G. Stefanidou, "Boundedness and asymptotic behavior of the solutions of a fuzzy difference equation," Fuzzy Sets and Systems, vol. 140, no. 3, pp. 523-539, 2003.

[10] G. Stefanidou and G. Papaschinopoulos, "A fuzzy difference equation of a rational form," Journal of Nonlinear Mathematical Physics, vol. 12, supplement 2, pp. 300-315, 2005.

[11] Q. H. Zhang, L. H. Yang, and D. X. Liao, "Behavior of solutions to a fuzzy nonlinear difference equation," Iranian Journal of Fuzzy Systems, vol. 9, no. 2, pp. 1-12, 2012.

[12] Q. Xiao and Q.-H. Shi, "Qualitative behavior of a rational difference equation $y_{n+1}=y_{n}+y_{n-1} / p+y_{n} y_{n-1}$," Advances in Difference Equations, vol. 2011, article 6, 2011.

[13] M. Pituk, "More on Poincaré's and Perron's theorems for difference equations," Journal of Difference Equations and Applications, vol. 8, no. 3, pp. 201-216, 2002. 


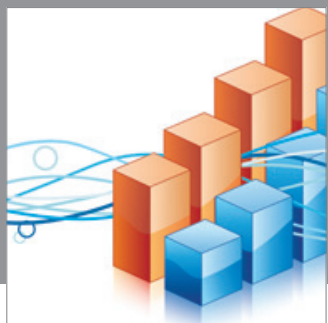

Advances in

Operations Research

mansans

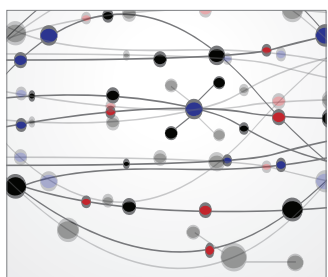

The Scientific World Journal
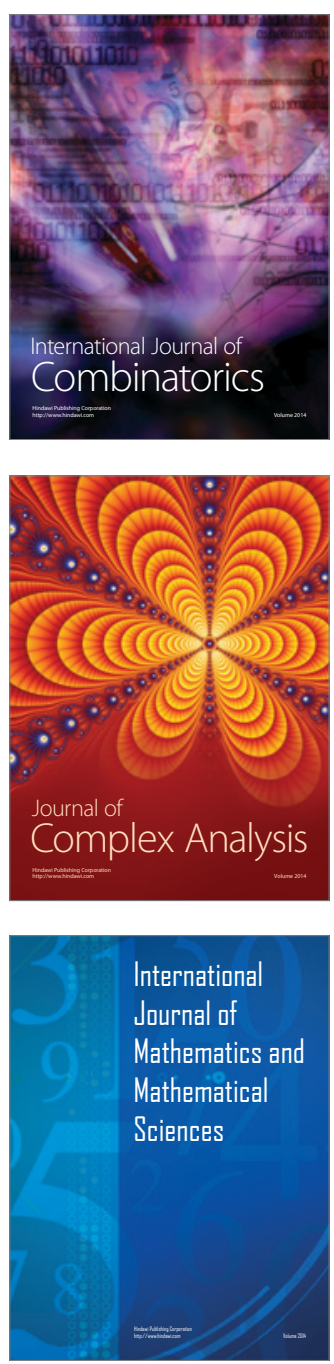
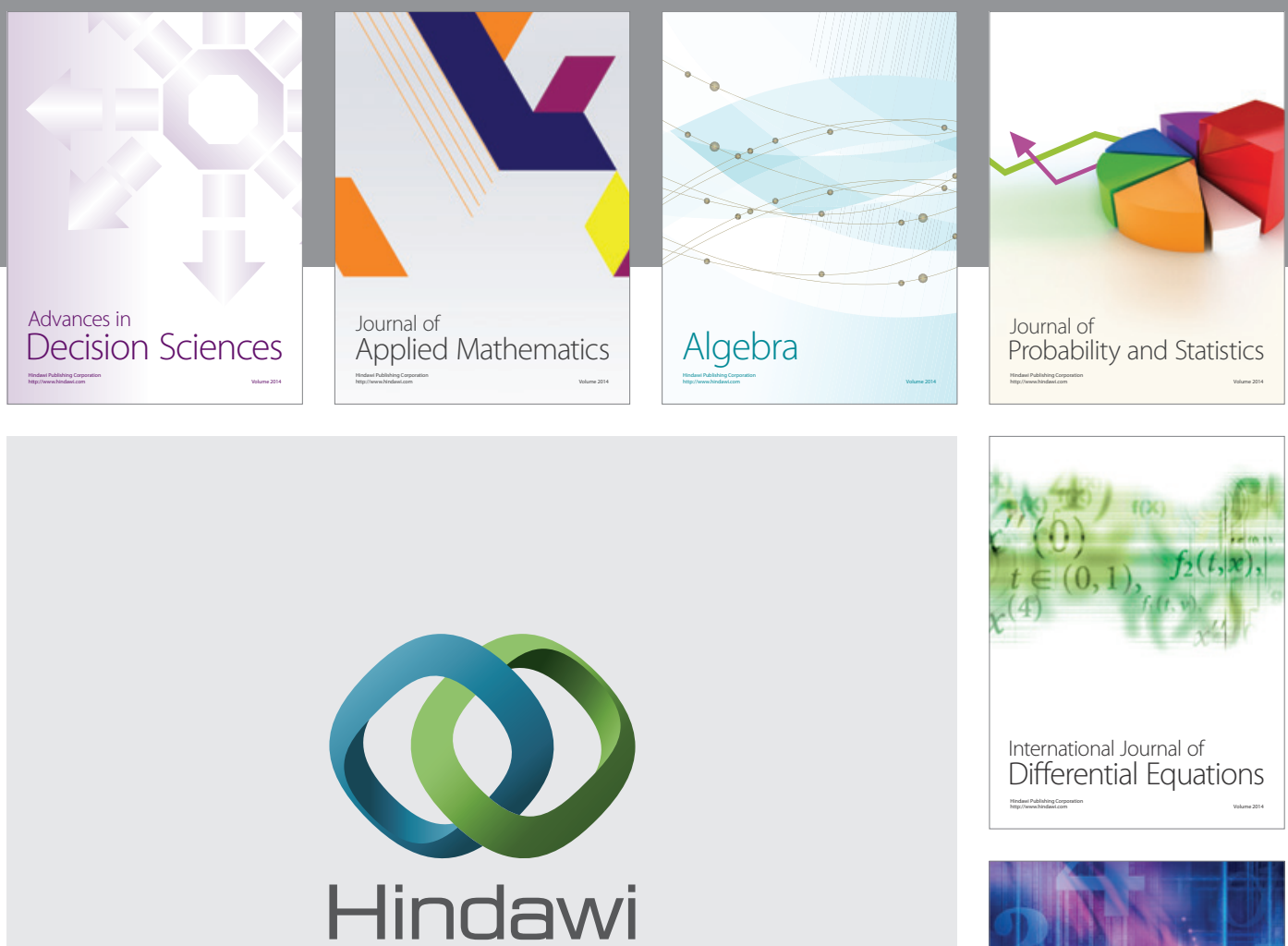

Submit your manuscripts at http://www.hindawi.com
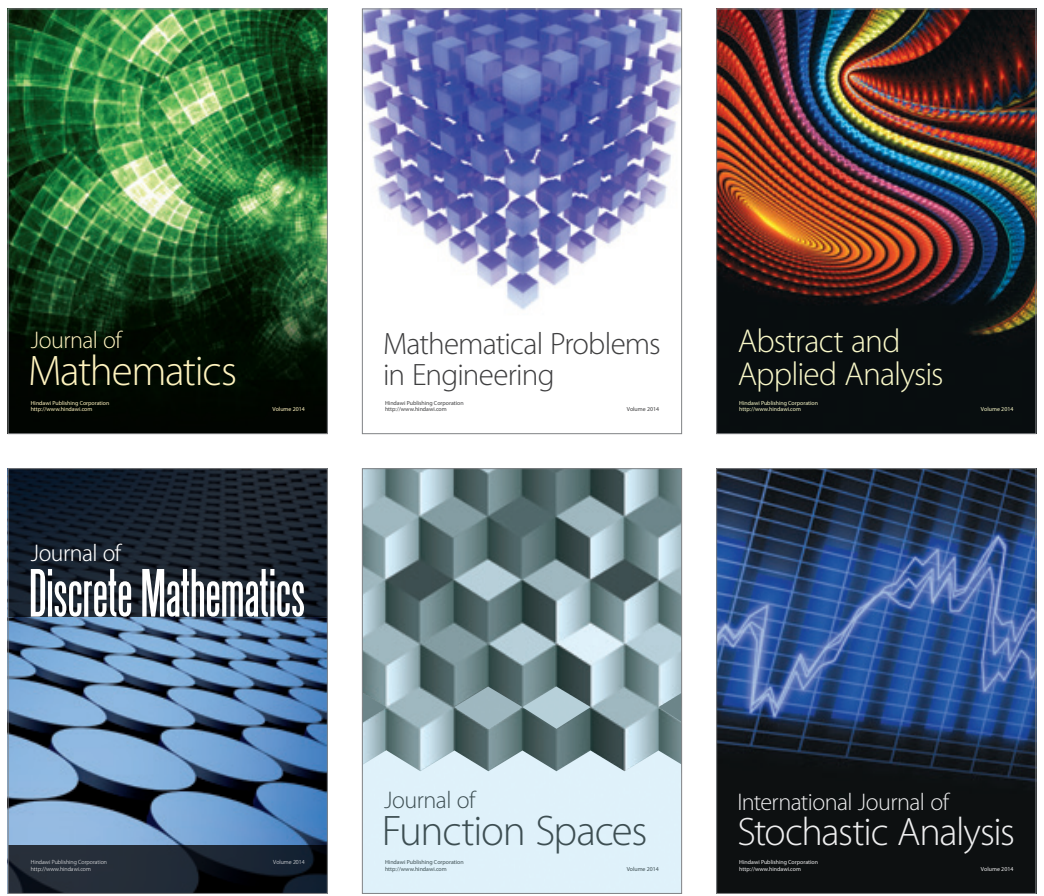

Journal of

Function Spaces

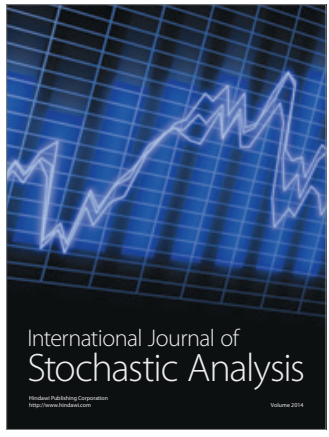

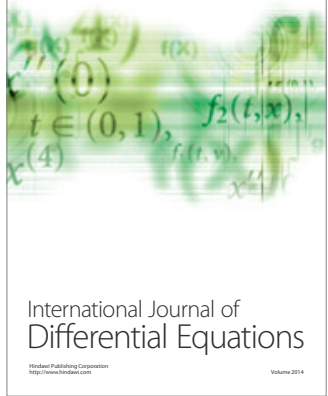
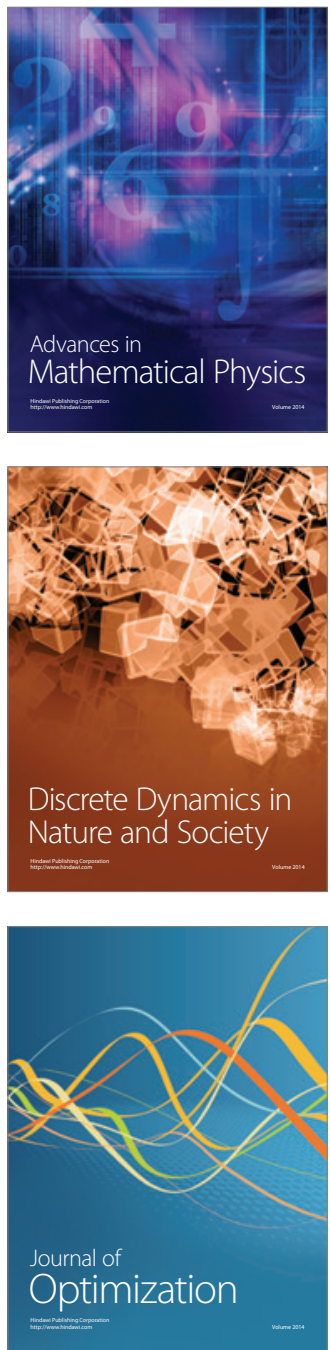